\title{
Embryonic epithelial Pten deletion through Nkx2.1-cre leads to thyroid tumorigenesis in a strain-dependent manner
}

\author{
Caterina Tiozzo ${ }^{1,2,3}$, Soula Danopoulos', Maria Lavarreda-Pearce', Sheryl Baptista', \\ Radka Varimezova', Denise Al Alam', David Warburton', Virender Rehan ${ }^{4}$, \\ Stijn De Langhe ${ }^{5}$, Antonio Di Cristofano ${ }^{6}$, Saverio Bellusci ${ }^{1,7}$ and Parviz Minoo ${ }^{1,2}$ \\ 'Developmental Biology Program, Saban Research Institute of Childrens Hospital Los Angeles, Los Angeles, \\ California 90027, USA \\ ${ }^{2}$ Department of Pediatrics, University of Southern California, Los Angeles, California 90027, USA \\ ${ }^{3}$ Department of Pediatrics, Nassau University Medical Center, 201 Hempstead Turnpike, East Meadow, New York \\ 11554, USA \\ ${ }^{4}$ Department of Pediatrics, Los Angeles Biomedical Research Institute at Harbor-UCLA Medical Center, Torrance, \\ California 90502, USA \\ ${ }^{5}$ Division of Cell Biology, Department of Pediatrics, National Jewish Health, Denver, Colorado 80206, USA \\ ${ }^{6}$ Department of Developmental and Molecular Biology, Albert Einstein College of Medicine, Bronx, New York, USA \\ ${ }^{7}$ Department of Internal Medicine II, Excellence Cluster Cardio Pulmonary System, University of Giessen Lung Center, \\ Klinikstrasse 36, Aulweg 130, 35392 Giessen, Germany
}

\author{
Correspondence \\ should be addressed \\ to $P$ Minoo or S Bellusci \\ Email \\ minoo@usc.edu or \\ saverio.bellusci@ \\ innere.med.uni-giessen.de
}

The authors and journal apologize for an error in the above paper, which appeared in volume 19 part 2, pages 111-122. In the author list, the first name and last name of Virender Rehan were transposed, and should have read as follows:

Caterina Tiozzo, Soula Danopoulos, Maria Lavarreda-Pearce, Sheryl Baptista, Radka Varimezova, Denise Al Alam, David Warburton, Virender Rehan, Stijn De Langhe, Antonio Di Cristofano, Saverio Bellusci, and Parviz Minoo 2012 Embryonic epithelial Pten deletion through Nkx2.1-cre leads to thyroid tumorigenesis in a strain-dependent manner. Endocrine-Related Cancer 19 111-122 (doi: 10.1530/ERC-10-0327). 